\title{
Application of a Computational Geometry Algorithm to Improve the Energy Consumption in a LoRaWAN Network
}

\author{
Esau Bermudez, Djamel F. H. Sadok \\ Center of Informatics (CIN) \\ Federal University of Pernambuco (UFPE) \\ Recife, Brazil
}

\begin{abstract}
Energy consumption in low power wide area networks such as LoRaWAN plays an important role in determining the lifetime of wireless sensor networks motes. In this paper, we examine the use of different battery technologies and the impact of the media access control (MAC) and physical (PHY) layers. Among the considered metrics in our sensor network scenario, we monitor energy efficiency and its impact on battery life and role for achieving better performance. The Jarvis algorithm its was used to examine the impact on the LoRa's MAC and physical layers, while changing their parameters such as the spreading factor, channel activity detection, code rate and channel frequencies. The results show both tested battery technologies discharge in a similar way. Lithium batteries exhibit increased resistance to discharge for a longer time and they are more cost profitable when executing the Jarvis algorithm.
\end{abstract}

\section{Introduction}

In the emerging universe of the Internet of Things (UIoT)[1], the LoRa radio technology and the LoRaWAN network architecture have become key players in low-power networks (LPWAN) [2]. The presence of new devices such as sensors and microprocessors for long-range communications allows the birth of a number of IoT applications such as smart metering, smart homes, and industry 4.0. We are moving to a world where all the things that surround us are connected [3]. Nonetheless, energy consumption remains a challenging technology where advances remain painfully slow to take place and as a result this is a research topic that remains highly interesting [4].

LPWAN, LoRa and its LoRaWAN protocol, have particularly attracted a great deal of attention due to their support for long-range and low-cost telecommunications [5]. The LoRa ends devices generally operate with batteries, especially in the case of the two LoRa device classes A and B. Class C type LoRa devices are expected to connect to the public energy grid. As a result, the design of Wireless Sensor Networks (WSNs) must explore all possible strategies for the reduction of power consumption [6].
In this context, it is necessary to take into account some important concerns. The adopted protocols at the medium access protocol (MAC) and physical layers must be carefully designed to save energy. For instance, the MAC protocol should avoid complex design of its channel access algorithm and must reduce packet collisions. Packet collisions result in unnecessary retransmissions [7]. It is clear that both MAC complexity and inefficient channel access cause a waste of energy and must be mitigated. Another case where energy is lost due to bad protocol design, would be during the Idle listening mode, in which the node remains listening to possibly receive traffic. That is, since the node does not know if it is going to receive a packet or not, it stays in the form of idle listening [8]. LoRa deals with this problem by allowing downlink reception only after a node has transmitted something.

In other words, a class A node only wakes up to transmit a packet when required by its application and will remain with its receipt channel open only for a small window of time. Some of the traditional Internet protocols such the Transmission Control Protocol (TCP) widely used today in the transport layer for end-to-end reliable exchange of information, were designed in a period where there was no concern with battery consumption as end devices were assumed to be connected to the grid. This is no longer the case with the presence today of more wireless and mobile devices than fixed ones. As a result, new transport protocols such as the Quick UDP Internet Connection (QUIC) have emerged for use in the Web. More particularly, QUIC establishes that When a server decides to terminate an idle session, it should not notify the client to avoid waking up the radio on mobile devices.

This is different from the behavior of transport protocols such as TCP that required that a wireless interface or radio must remain switched on all the time even when it is not receiving packets.

Class B LoRa nodes are different in that they schedule their transmissions periodically over time whereas class $\mathrm{C}$ devices are supplied from the public energy grid and therefore are not concerned with energy saving. Nonetheless, nodes from all classes must always hen-operating listen to the radio interface 
to determine if a given information is destined for them. Thus, the LoRa control packets also consume energy, and the receiving and transmitting stations of these packets add to energy consumption [9].

One point to take into account is adaptability, which results from the dynamics of the network. The basic idea is to put a node to sleep if there is no activity scheduled for that node. However, it is necessary to coordinate the nodes when they go to sleep so that they acquire a certain synchronism, and thus avoid wasting energy [10]-[12].

Based on the importance of LPWANs for different IoT scenarios, the authors in [13] show the strict reliability and energy efficiency requirements imposed by IoT networks. The study examines both existing short-range industrial solutions and longrange communication technologies and compare these in terms of scalability, radio coverage and energy efficiency.

According to [14], LPWANs have so far been dominated by two widely adopted solutions; these are, the ultra-narrow band technology by Sigfox and the Chirp Spread Spectrum (CSS) technology from Semtech Corporation [15]. Both solutions are then evaluated in terms of coverage and power consumption. LoRaWAN is being widely deployed due to its competitiveness in many IoT uses cases and applications [16].

In the absence of $5 \mathrm{G}$ machine to machine communications (MMC), many works go further and argue that LoRaWAN is currently the best alternative in terms of cost, battery life and energy efficiency. Current 4G NB-IoT is outstanding with respect to quality of service (QoS), latency, and reliability [17]. The authors in [18] tested signal propagation over a range of detection intervals while monitoring consumption. Cost may be a limiting factor as $4 \mathrm{G}$ devices require service subscription and the use of a Subscription Identity Module (SIM) card.

In [18], the authors also considered that LoRaWAN is an alternative that has become attractive for all companies due to its low cost of implementation and its advantage in technological infrastructure efficiency, which has increased its visibility in the market, and it has made it more important due to all its benefits in quality of service (QoS), latency and reliability as mentioned in [17].

On the other hand, Sigfox technology is often preferred when the communication range is the main design criterion. This technology is known to offer greater radio coverage [19], [20]. The work concludes that a large coverage area and longer battery life of end devices can be guaranteed in this case. Similarly to our work, the impact of battery technology or chemical composition on LoRaWAN performance optimization was evaluated in [21], [22].

In this paper, a LoRa network of four nodes is presented, in which the energy consumption performance in the nodes is compared using Jarvis algorithm. To evaluate battery life performance, the CupCarbon simulation software was use. The sensors that compose the network are the SX1272/73 devices manufactured by the Semtech Corporation. The conducted comparison takes into account two types of batteries, one of lithium and the other one of nickel. We sought finding the best performance in the discharge time of the battery while using the sensors to transmit data packets.

This paper is organized in five sections. Taking into account the analysis of the LoRaWAN protocol and the battery discharge analysis, the first section performs a discussion of the LoRaWAN network protocol and LPWAN technology. In the second section the problem statement is made, the concepts and most of the formal equations showing energy consumption, time in air, among others, are discussed. In the third section the simulation of the sensor network and batteries operation, their discharge and useful life are presented. In fourth section, the results are drawn and discussed. Finally, fifth section concludes our work and points to possible future work.

\section{Problem statement}

\subsection{LoRaWAN}

Communication in LoRaWAN networks [21] is subject to a number of configuration parameters such as the Spreading Factor (SF), Code Rate (CR) and channel frequency. LoRa uses an SF between 7 and 12. A larger SF increases the time on air therefore improving the communication range at the detriment of the data rate which is reduced. The SF is expressed mathematically in (1), where it is possible to observe the logarithmic relationship between the symbol rate (Rs) and the chip rate $(\mathrm{Rc})$.

$$
S F=\log _{2} \frac{R_{c}}{R_{S}}
$$

One major factor for taking in account is the packet time. It is the time taken to send a packet to the sub-band for subsequent transmission and is indicated in (2). Note that the longer this transmission time, the greater the range.

$$
T_{\text {Packet }}=T_{\text {Preambles }}-T_{\text {Payload }}[s]
$$

Where $T_{\text {Preambles }}$ contains the preamble time to transmit and the $T_{\text {Payload }}$ is the total time required to transmit a payload which are expressed in (3) and (4).

$$
\begin{gathered}
T_{\text {Preamble }}=\left(n_{\text {Preambles }}+4.25\right) T_{S} \\
T_{\text {Payload }}=P L_{\text {Symb }} * T_{s}
\end{gathered}
$$


With $n_{\text {Preambles }}$ the length of programmed preamble, PL the number of payload symbols and Ts the time required to transmit a single symbol, which is expressed as:

$$
T_{S}=2 S F \frac{1}{B W}
$$

Also, it is important to consider the time during which the channel is not available for transmission which is known as free time (Toff), and its mathematical representation is shown in (5). If the channel is not available at the time of transmitting, the end node must have to wait a Toff interval before scheduling the subsequent transmission.

$$
T_{\text {off }}=\frac{\text { TimeOnAir } 1}{\text { DuttyCycle }_{\text {subband }}}-\text { TimeOnAir }
$$

There are limitations that establish how long the transmitter can be activated or the maximum time it can be transmitting. LoRaWAN imposes a duty cycle limitation for each sub-band (5). Each time a frame is transmitted in a given sub-band, the time of the broadcast and the on-air duration of the frame are recorded for this sub-band [18].

For the US902-928 (US operating version of LoRa standard), the LoRaWAN Regional Parameters 1.0.2 Rev B as used by The Things Network (TTN) community network, describe that Federal Communications Commission regulations impose a maximum dwell time of 400 milliseconds on uplinks. That makes data rate zero (DR0) use spreading factor (SF) 10, as for SF11 Bandwidth (BW) $125 \mathrm{kHz}$ and SF12 BW $125 \mathrm{kHz}$.

The minimum LoRaWAN overhead would already need more time on air than ever allowed [15]. Similarly, to any TTN region, the TTN Fair Access Policy allows for at most 30 seconds uplink airtime and 10 downlink messages (including ACKs for confirmed uplinks) per device, per 24 hours. In other words, it is important to control the duty cycle by reducing its value to ensure that a user may not remain for a long time monopolizing a LoRa channel and that other have a chance to transmit and do not wait to a long time to gain access to radio channels.

Equally important is the concept of the Channel Activity Detection, which is designed to detect a LoRa preamble on the radio channel with the best possible energy efficiency. LoRa devices of type A and B are equipped with a battery. For this reason, the duty cycle operation should be analyzed to achieve better battery life. During measurements, nodes in the network periodically go to sleep before their next transmission cycle. Therefore, the detection interval (IDect) is defined as the pause time between nodes and it is expressed in (6) [23].

$$
I_{\text {Dect }}=I_{S}+2 I_{S W}
$$

Where $I_{S}$ is the amount of time during which the nodes remain in sleep mode and $I_{s w}$ is the time that the nodes take to switch between active and sleep mode. The short detection interval helps to detect the anomaly in early stages; it also causes a reduced battery life. For this reason, it could be analyzed from the economic cost, functional and lifetime of the network. Therefore, the efficiency of the system would be at stake.

\subsection{Computational Geometry}

The Computational geometry systematic is the study of algorithms and data structures for geometric objects, included computer graphics, computer vision and others [24].Next, it is describe the one we use in this study, namely, the Jarvis algorithm. This is a gift wrapping algorithm used for computing the convex hull of a given set of points.

Jarvis Algorithm: Jarvis march computes the $\mathrm{CH}(\mathrm{Q})$ by a technique known as gift wrapping or package wrapping. Consider first, a base point P0 (S1Detection) is selected. This is the point with the minimum y-coordinate provided in these scenarios with the LoRaWAN protocol. Select leftmost point in case of tie. The next convex hull vertices P1 has the least polar angle w.r.t. the positive horizontal ray from P0. Measure in counterclockwise direction. If tie, choose the farthest such point. Vertices P2, P3, ... Pk are picked similarly until $\mathrm{Yk}=\mathrm{Ymax} . \mathrm{Pi}+1$ has least polar angle w.r.t. positive ray from $\mathrm{P}_{0}$. If tie, choose the farthest such point. Worst case occurs when $\mathrm{O}(\mathrm{n})$ points lie on the convex hull i.e., all points lie on the circle.

\subsection{Energy Consumption}

In real applications, many types of batteries can be considered, where their useful life depends on climatic variations and the type of node considered sensor. In a simulation of a wireless sensor, the useful life of the network is conditioned by the battery status of each node. That is why correctly modeling the battery becomes important to obtain successful results of a WSN operation [23].

One way to assess the status of the battery is through equation (7).

Battery Life $[h]=\frac{\text { Battery Capacity }[\mathrm{mAh}]}{\text { Charging Current }[\mathrm{mA}]}$ 
Table 1. Sensors location

\begin{tabular}{|c|c|c|c|}
\hline Node & $\begin{array}{l}\text { Latitude / } \\
\text { Longitude }\end{array}$ & $\begin{array}{c}\text { Distance } \\
{[\mathrm{Km}]}\end{array}$ & $\begin{array}{l}\text { Free } \\
\text { Space } \\
\text { Loss } \\
{[\text { dB] }}\end{array}$ \\
\hline 1 & $\begin{array}{l}8.051 .549 .216 .211 . \\
980 /- \\
3.494 .926 .929 .473 . \\
870\end{array}$ & 0.149 & $\begin{array}{l}75,0361 \\
331\end{array}$ \\
\hline 2 & $\begin{array}{l}8.052 .760 .244 .966 . \\
250 / \\
34.949 .880 .838 .39 \\
4.100\end{array}$ & 0.149 & $\begin{array}{l}75,0361 \\
331\end{array}$ \\
\hline 3 & $\begin{array}{l}8.054 .035 .008 .159 . \\
960 /- \\
3.495 .019 .197 .463 . \\
980\end{array}$ & 0.146 & $\begin{array}{l}74,8594 \\
6484\end{array}$ \\
\hline 4 & $\begin{array}{l}8.054 .959 .209 .124 . \\
860 /- \\
3.495 .118 .975 .639 \text {. } \\
340\end{array}$ & 0.151 & $\begin{array}{l}75,1519 \\
4667\end{array}$ \\
\hline
\end{tabular}

The average load (A) in the state of transmission, sleep and radio switching time can be estimated taking into account the current plotted in different monitoring modes and the length of time that a device remains in a certain state. They can be expressed as in (8), where Ttx is the time when a node is transmitting and Ctx is the average current consumed in transmission mode [18].

$$
\bar{A}=\overline{C_{t x}} T_{t x}
$$

\section{Simulation}

This section provides a breakdown of the results and conducted analysis of LoRaWAN using the simulation tool CupCarbon. Without loss of generality, a simple LoRaWAN network composed of four sensors in an almost linear arrangement was proposed. The network was located between the NIATE (Integrated Nuclei of Activities of Ensino) and the CIn (Computer Center) of the UFPE Federal University in Recife, Brazil.

\subsection{LoRaWAN Network}

The network has specific coordinates, node source and node destination (Longitude: 3.494.926.929.473.870, Latitude: 8.051.549.216.211.980) and (longitude: -3.495.118. 975.639.340 latitude: 8.054.959.209.124.860) and node destination respectively. The location and parameters of the nodes are shown in Table 1 and the sensor parameters used in the Cupcarbon simulation tool and LoRa are presented in Table 2.

In this study, the characteristics of the nodes are represented with a transceiver set using CupCarbon.
All peripherals are powered at the same voltage level equal to $3.30 \mathrm{~V} \mathrm{Li}$-Ion and 1,4 V for $\mathrm{NiCd}$ batteries.

Table 2. Sensors parameters

\begin{tabular}{|l|l|}
\hline Parameter & Value \\
\hline Radius & $20 \%$ \\
\hline Energy Max & $19160 \mathrm{~J}$ \\
\hline Data Rate UART & 9600 bits \\
\hline Drift (Sigma) Clock Freq & $3.0 \mathrm{E} 5$ \\
\hline Node Radius & 130.0 \\
\hline PL (Power Transmission) & 100.0 \\
\hline Sending energy consumption (ETx) & $5.92 \mathrm{E}-5$ \\
\hline ERx: Receiving energy consumption & $2.86 \mathrm{E} 5$ \\
\hline Sleeping Energy & $1.0 \mathrm{E} 7$ \\
\hline Listening Energy & $1.0 \mathrm{E} 6$ \\
\hline Spreading Factor & 7 to 12 \\
\hline Channel & 0 \\
\hline
\end{tabular}

\subsection{Sensor Node}

The hardware of a sensor node can be broken down into five main parts: the processor, the sensor, the storage module, the transmitter and the power supply. All the elements of the sensor are using the power supply simultaneously. The SX1272 transceivers feature the LoRa long-range modem that provides ultra-long-range and wide-spectrum communication and high interference immunity while minimizing current consumption.

Considering the sensor network, in Figure 1 with the network nodes S1, S2, S3 and S4. Its energy reception consumption and transmission consumption are expressed by called PRx (9) respectively.

Table 3. Simulation results

\begin{tabular}{|c|c|c|c|c|c|}
\hline $\begin{array}{l}\text { Execution } \\
\text { Time[s] }\end{array}$ & $\begin{array}{c}\text { Msg } \\
\text { Sent }\end{array}$ & $\begin{array}{c}\text { Msg } \\
\text { Received }\end{array}$ & ACK & $\begin{array}{c}\text { Msg } \\
\text { Lost }\end{array}$ & $\begin{array}{c}\text { Total } \\
\text { msg } \\
\text { Sent } \\
+ \text { Rec }\end{array}$ \\
\hline 100 & 209 & 208 & 208 & 1 & 417 \\
\hline 300 & 598 & 597 & 597 & 1 & 1195 \\
\hline 700 & 1388 & 1388 & 1388 & 0 & 2776 \\
\hline
\end{tabular}


Table 4. Batteries types

\begin{tabular}{|c|c|c|}
\hline Parameters & NiCd & Li-Ion \\
\hline $\begin{array}{c}\text { Relative } \\
\text { Capacity }\end{array}$ & $1.4 \mathrm{~V}$ & $3.7 \mathrm{~V}$ \\
\hline Cycle Life & $\begin{array}{c}500-1000+ \\
\text { cycles }\end{array}$ & $1200+$ cycles \\
\hline $\begin{array}{c}\text { Operating } \\
\text { Temperature }\end{array}$ & -20 to $50^{\circ} \mathrm{C}$ & -10 to $50^{\circ} \mathrm{C}$ \\
\hline
\end{tabular}

$P_{\text {elec }}$ is the power consumption in the node circuit, $K$ is the constant that represents the minimum power with success, $\alpha$ is the attenuation coefficient with distance and $r$ is the distance from source to destination, and $i$ represents the hops between nodes [25]. In order to establish the total consumption of transmission and reception with over a single hop, the equation (10) is applied.

$$
\begin{aligned}
P_{T x} & =P_{\text {elec }}+K r^{\propto}, \\
P_{T 1} & =P_{T x 1}+P_{R x 1}, \\
& =P_{\text {elec }}+K r^{\propto}+P_{\text {elec }}, \\
& =2 P_{\text {elec }}+K r^{\propto} .
\end{aligned}
$$

The execution of the algorithm in the CupCarbon software provides a triangulation between its nodes and in turn a hop between nodes S1 to S3 to reach the destination node as it is shown in Figure 1.

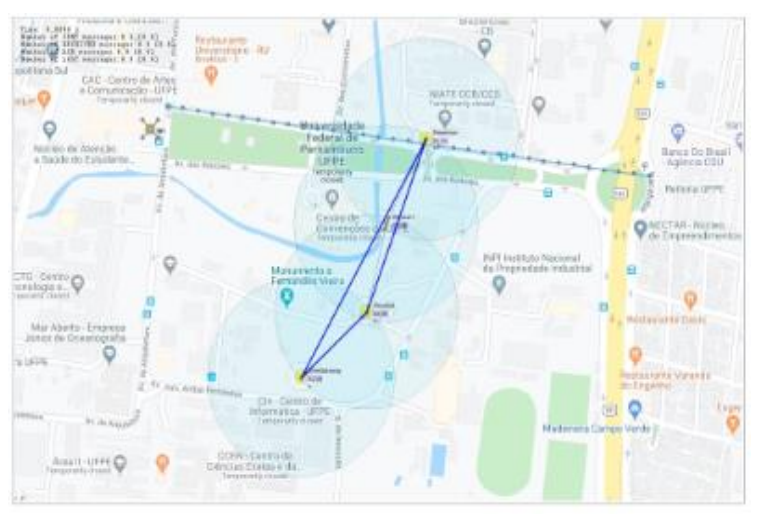

Figure 1. Sensors network distribution

This triangulation between its nodes provides the data that is subsequently analyzed for the purposes of the analyzing energy consumption of the network, taking into account that this consumption was calculated according to the self-recharge that the battery suffers when it stops being used.

According to the triangulation between the nodes carried out by the execution of the algorithm, three observation times are established in the execution of the algorithm, resulting in the data from Table 3, for subsequent analysis, regarding the energy consumption of the network. On the other hand, it was observed that the discharge of the Nickel battery over time, manages to reduce the difference of days with respect to that of Lithium with the Jarvis algorithm. This same is evidenced in Table 3, where the greater better performance runtime.

\subsection{Batteries}

Two types of batteries were used to calculate their performance and thus establish which one is the best to use in this simulation for a possible project. This study considers two types of batteries, namely, Lithium and Nickel batteries. The battery parameters are found in Table 4.

\section{Results and discussion}

In this section, it is present the results obtained from the simulation according to the parameters established throughout the document by the adopted models and equations described above.

As can be seen in Figure 2, the reddish color which is the propagation spectrum of the nodes and in turn the possible routes that can be established between the nodes. These routes are marked by the gray lines shown in the aforementioned Figure 2, which are tested when executing the algorithm.

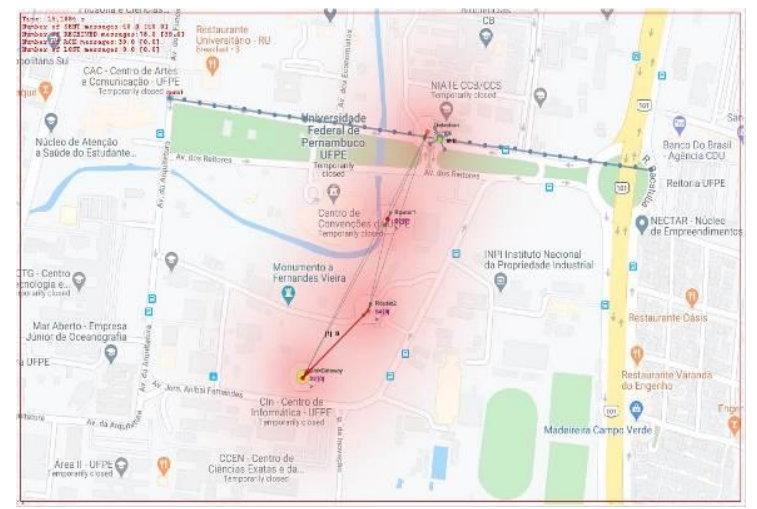

Figure 2. Spectrum signal

On the other hand, the spectrum signal shown in Figure 2, is the propagation of the signal with the node's radio configuration at $20 \%$, according to the configuration Table 2.

This propagation could be higher if the percentage of the node's radio configuration were increased, this percentage when increased can reach a greater range in terms of its distance, and this must be taken into account according to the needs of the network configuration. That is why the benefits that can be found in these types of sensors and LoRa technology 
and its LoRaWAN protocol help us to provide customized solutions on many occasions.

According to Figure 3 that corresponds to the percentage of consumption of the sensors is set below 0.0250 of energy in Jules. Favoring the performance of the sensors battery.

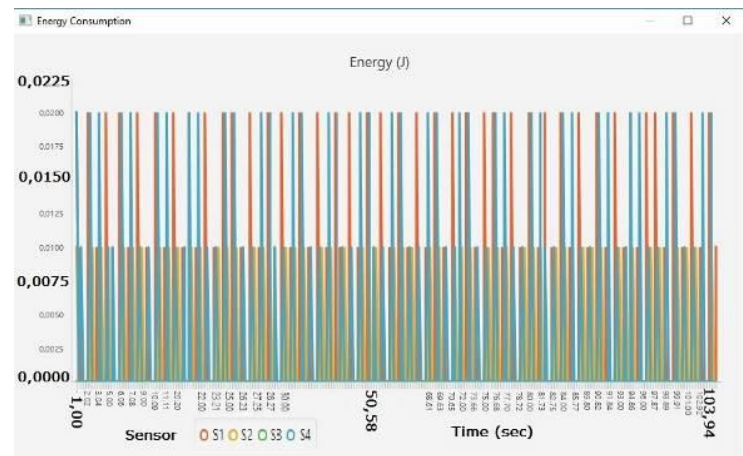

Figure 3. Consumption percentage

Each sensor transmits according to the request, that is, it wakes up and attends to the request for transmission of the requested packets, this in order to minimize battery consumption and extend its useful life. The Jarvis algorithm adapts the network according to the triangulation generated by its mathematical structure. Since what is desired is to start at a base node, which in this case is S1Detection, until reaching the furthest node. Taking into account that locates all the nodes of the network and, according to the position of each one, performs the triangulation shown in Figure 4.

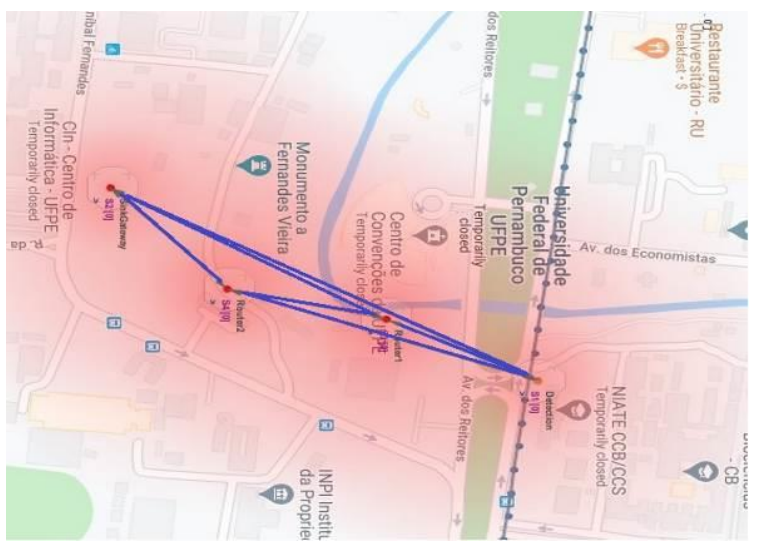

Figure 4. Triangulated routes

The battery consumption as shown in Figure 5 established during the battery discharge time when transmitting and therefore when executing the Jarvis algorithm, is 2.50 Jules as a reference. This summation of discharges generated by the sensors in the network is below $0.5 \%$ of the total battery charge. It is also evidenced as shown in Figure 5, that node S3 (green line) remains without request, this due to the configuration adopted by the algorithm, that is why this node could serve as a backup in an eventual network anomaly when executing the algorithm to generate another communication path between the nodes.

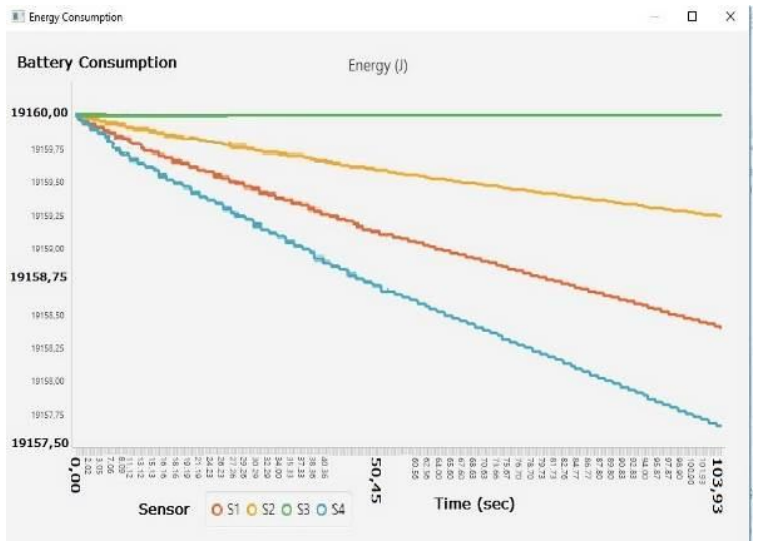

Figure 5. Battery consumption

By observing the consumption behavior of a sensor individually, as shown in Figure 6, the percentage of discharge can be seen at the time of executing the algorithm, which is 0.0200 Jules.

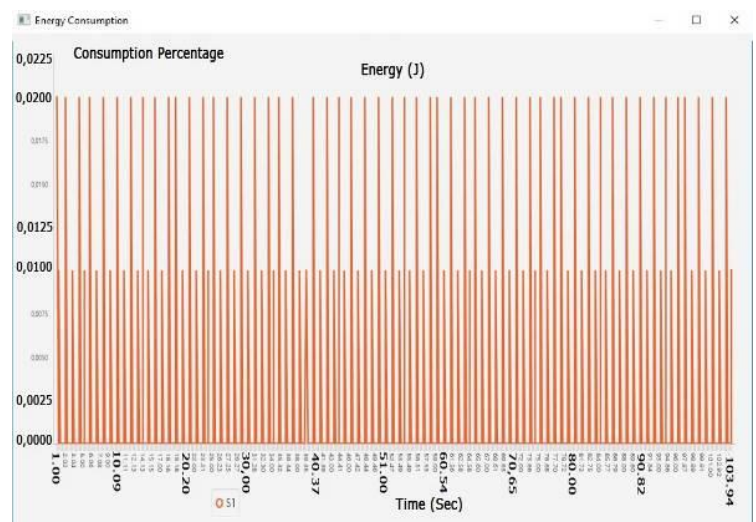

Figure 6. Node consumption

When the values generated by the .cvs file Figure 5 are plotted, it is observed that nodes S1 and S4 intersect at times and downloads, that is, $x y$ vectors where $x$ is time and $y$ is energy consumption thus: (5.06; 191.599.775), (7.06; 191.599.702) and (15.13; 191.599.550). It is interesting to see how Figure 7, is the same as the execution of the algorithm in the program, that is, Figure 8 . Showing the same 
discharge pattern only with the aforementioned crosses.

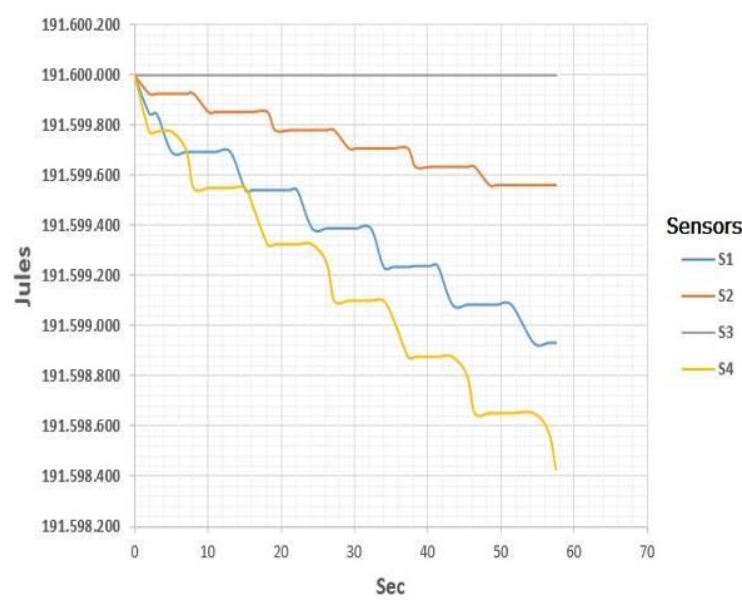

Figure 7. Intercept nodes S1 and S4

This new test was carried out in the $\mathrm{R}$ program as verification of the data and its congruence with the previous ones. At the time of battery discharge, the crossing of nodes S1 and S4 was analyzed, which is given by the request time at the beginning of the transmission, since S1Detection (node S1) is the main node where it is executed the triangulation of the algorithm, which requires higher consumption at certain request times in sending and receiving packages. This can be seen in Figure 8 , where the arrows point showing these moments.

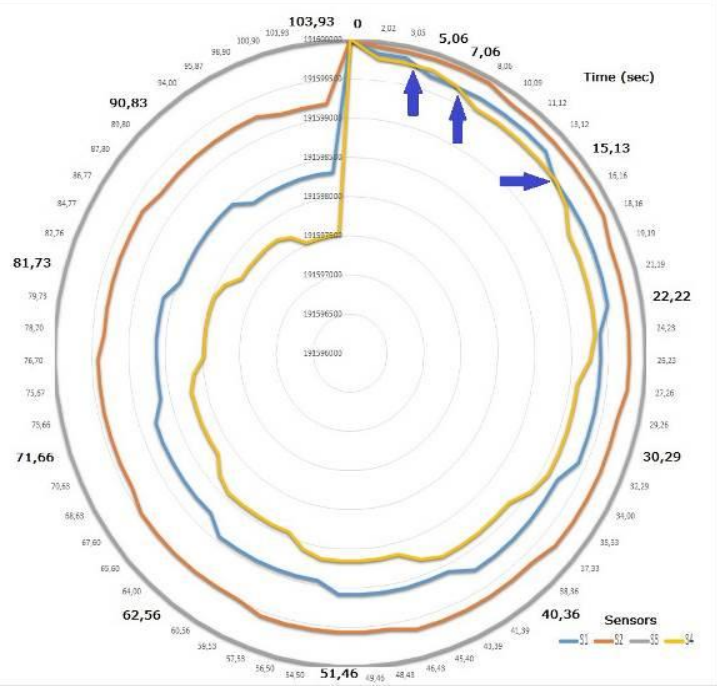

Figure 8. Intercept time on nodes S1 and S4

In Figure 7 and Figure 8, it can be seen that node S3 (gray line) does not participate in the transmission and therefore remains without energy consumption, as previously stated in the discussion.
The behavior of Time on air (ToA) with respect to the bandwidth used is presented in Figure 9, represented in (1) and (6). A decrease in airtime was observed as bandwidth increased, which is an intuitive result. Based on this, it was decided to use the $125 \mathrm{kHz}$ LoRa channel bandwidth setup.

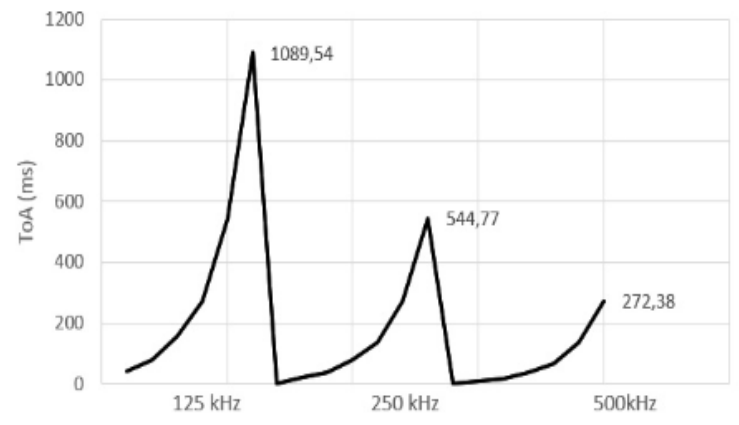

Figure 9. ToA (ms) vs Bandwidth $(\mathrm{kHz})$

This bandwidth allowed us to obtain a better result in the simulation and to also focus on the preliminary results regarding the use of the battery in this bandwidth.

In the CAD it is evident that in the course of the consumption load, the $I_{s}$ at a certain point of the curve shows a decrease. The IDect could give us an idea of failure or anomalies in the network causing a short battery life, in this way it was possible to take into account both the economic cost and the useful life of the battery. This occurred with both batteries presenting the same trend, ending the battery consumption charge as seen in Figure 10.

The duty cycle established during the airtime that each packet takes to be transmitted is represented according to the duration of the ToA. It allows a successful delivery according to (2), (3) and (4).

Figure 11, shows how it will affect battery consumption. When analyzing the useful life of the batteries in (8), it was find that the Lithium battery has a more optimal performance than the Nickel battery, according to the number of bytes used as a workload mode in each of them. Observe

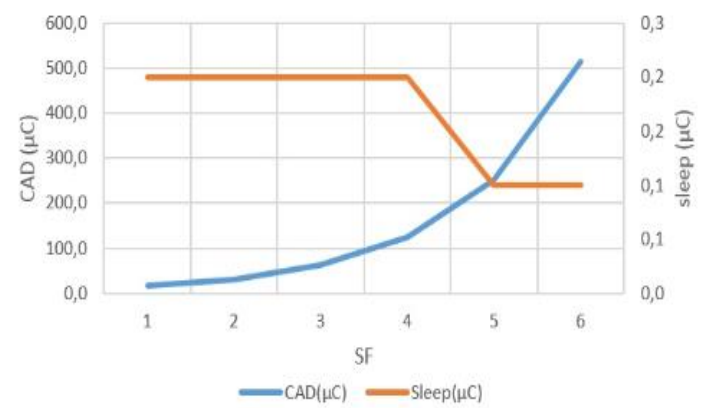

Figure 10. CAD vs Sleep 
that in duration per days the Lithium battery with respect to the Nickel battery shows greater performance during the simulation. In the lowest percentage of days, Figure 12 shows that the difference is significant for the prolongation of the life for this type of sensors (SX1272/73) and therefore the network.

When the energy per days of the batteries and the duty cycle are analyzed, it was deduced that the higher the duty cycle is, the more energy the batteries require to meet the needs and demands of the duty cycle. This caused the batteries to incur a greater energy drain to maintain the parameters required in the transmission of packets from the source node to the destination node, according to (9). Since the nodes must stay for a longer time sending information this caused them to be awake for longer periods as shown in Figure 13. When transmitting, it is observed that the longer the transmission time, the greater the discharge.

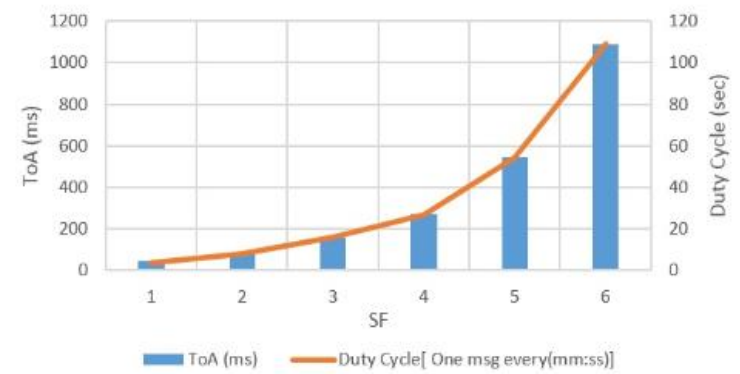

Figure 11. ToA vs Duty cycle

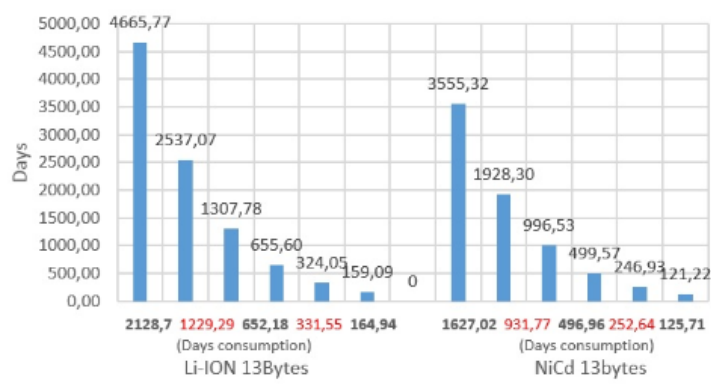

Figure 12. Estimation of batteries life (days) Lithium vs NiCd

The discharge of both batteries is evident in Figure 14 , but the Nickel battery suffers a greater discharge during the same time determined for both batteries, that is, its useful life was shorter than that of the Lithium battery. On the other hand, it could be observed in the image that the performance of the lithium battery was above that of the Nickel battery at the time of transmitting $\mathrm{P}_{\mathrm{Tx}}$ and upon receiving $\mathrm{P}_{\mathrm{Rx}}$ packets signal over a single hop according to (10). Overall, this makes the power consumption given by Pelec more efficient in both batteries, in a certain time established by the ToA for sending and receiving packages, reflecting the behavior of (6) and (8).

Energy consumption costs for a hop between nodes were low according to (10). It was observed in Figure 15, it can be concluded that the current consumed by the batteries in the concluded that the current consumed by the batteries in the process of sending and receiving packages in each node was very low,

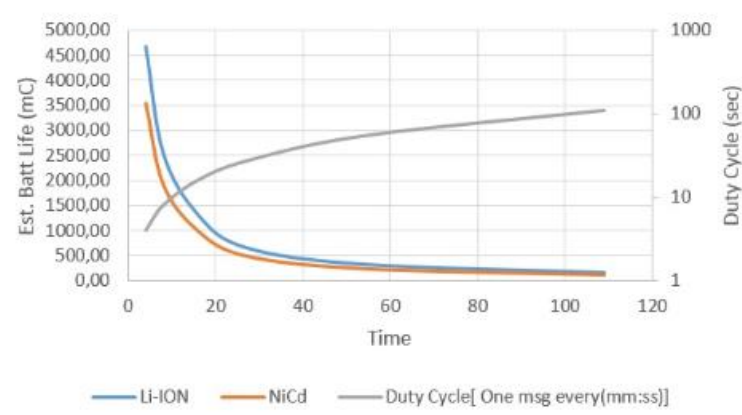

Figure 13. Energy per day vs Duty cycle

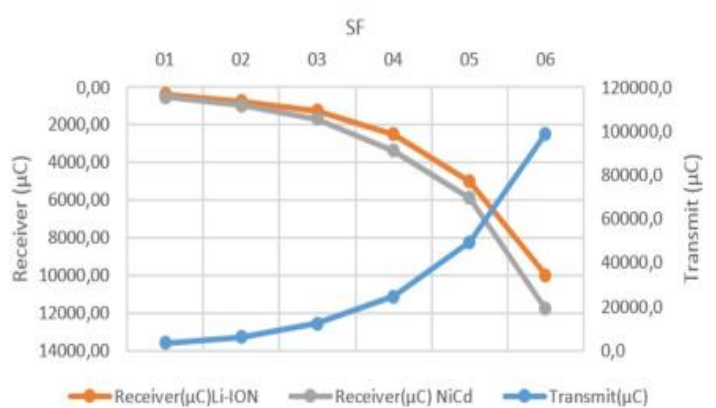

Figure 14. Transmitter vs Receiver

with an average of 0.0250 Jules, which implies an energy consumption below $0.5 \%$ of the energy available at the sensor node. This result shows that although both batteries have the same discharge behavior, that is, when they start to discharge, their useful life is different, with the Lithium battery having a longer duration compared to the Nickel battery in Figure 13.

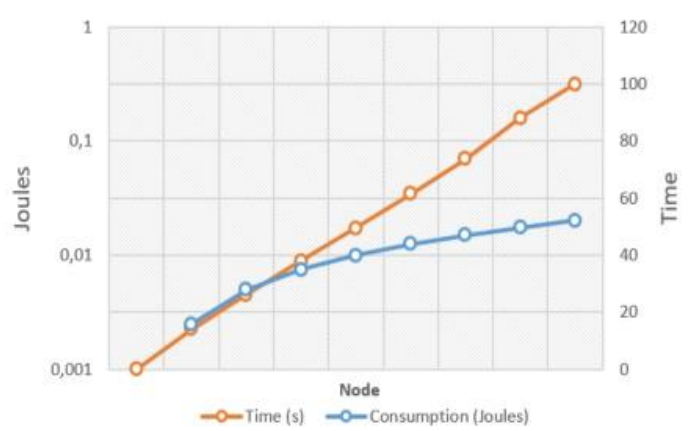

Figure 15. Node consumption 
On the other hand, the total battery charge and the mean current is the same for both batteries and shows a decrease in the course of the days in which they are used. This causes the state of battery life to decrease as the current increases and the load of the battery decreases in equal percentage for both batteries. It is dependent of the duty cycle of each one. This is where storage capacity takes on a significant advantage between the two batteries.

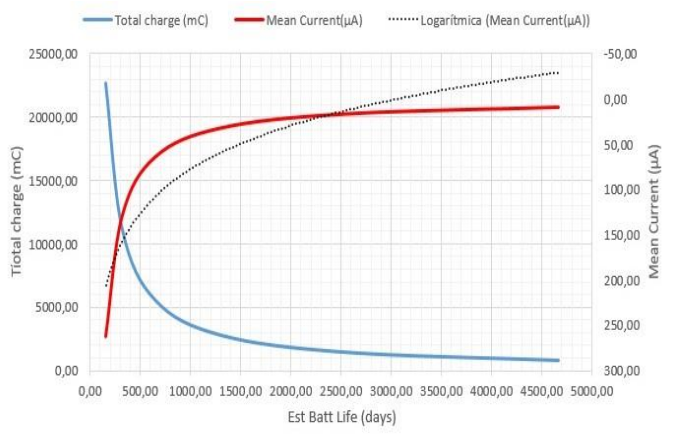

Figure 16. Total charge vs Mean current

As long as the storage capacity is greater, a better performance response over time can be obtained. The Lithium battery showed better performance over time than the Nickel battery as seen in Figure 16. This other aspect comes into consideration in terms of the durability and useful life of each battery.

\section{Conclusions}

In the execution of the algorithm, the LoRa network and its LoRaWAN protocol present alternate routes that can be used in the event of communication failures as a viable option in the network, although this new route must be parameterized in a certain case.

Regarding the duration of the battery in the motes, the values obtained comply with the characteristic of high durability, although the values are conditioned by the consumption parameters established in the configuration of the nodes registered in Table 2, where there is only Take into account the parameters of the LoRa SX1272/73 chip.

The types of batteries used in this evaluation were the Lithium battery and a Nickel battery. The durability due to the state of charge of the Lithium battery surpasses that of the Nickel battery, since the Lithium battery has a higher voltage and a better capacity than the Nickel battery. This aids in the modeling of a sensor network, and the arrangement of the speckles for better energy efficiency. On the other hand, it was observed that the discharge of the Nickel battery over time, manages to reduce the difference of days with respect to that of Lithium with the Jarvis algorithm.

The consumption charge for both batteries at the time of transmission behaved in the same way in both cases. The consumption charge of the receiver in the Lithium battery was lower than in the Nickel battery, therefore this generates a faster wear on the part of the Nickel battery. The total charge and energy per day of the batteries behaved the same in both cases. Variation in the state of life of the battery was evidenced, this was given by its voltage (V) and its capacity (mAh) respectively. Where the lithium battery showed greater prolongation of useful life over time.

In future work, the effect of the LoRaWAN P2P scheme to negotiate reception of packets could be analyzed to improve the performance of the network, its communication between nodes, its useful life and therefore the life of the battery. In addition, it is planned to evaluate the impact of temperature on the behavior of the batteries under different conditions.

\section{References}

[1] E. B. Sanchez and D. F. H. Sadok, "LoRa and LoRaWAN Protocol Analysis Using Cupcarbon," in Telematics and Computing, 2020, pp. 352-376.

[2] IEEE, "IEEE Standard for Low-Rate Wireless Networks - Amendment 5: Enabling/Updating the Use of Regional Sub-GHz Bands," IEEE Std 802.15.4v-2017 (Amendment to IEEE Std 802.15.4-2015, as Amend. by IEEE Std 802.15.4n-2016, IEEE Std 802.15.4q-2016, IEEE Std 802.15.4u-2016, IEEE Std 802.15.4t-2017), pp. 1-35, 2017.

[3] M. A. Ertürk, M. A. Aydın, M. T. Büyükakkaşlar, and H. Evirgen, "A Survey on LoRaWAN Architecture, Protocol and Technologies," Futur. Internet, vol. 11, no. 10, pp. 216-250, 2019.

[4]D. Zorbas, K. Abdelfadeel, P. Kotzanikolaou, and D. Pesch, "TS-LoRa: Time-slotted LoRaWAN for the Industrial Internet of Things," Comput. Commun., vol. 153, pp. 1-10, 2020, https://doi.org/10.1016/j.comcom .20 20.01.056.

[5] J. P. Queralta, T. N. Gia, Z. Zou, H. Tenhunen, and T. Westerlund, "Comparative Study of LPWAN Technologies on Unlicensed Bands for M2M Communication in the IoT: beyond LoRa and LoRaWAN," Procedia Comput. Sci., vol. 155, pp. 343-350, 2019, https://doi.org/10.1016/j.procs. 2019.08.049.

[6] K. E. Nolan, W. Guibene, and M. Y. Kelly, "An evaluation of low power wide area network technologies for the Internet of Things," in 2016 International Wireless Communications and Mobile Computing Conference (IWCMC), 2016, pp. 439-444.

[7] R. B. Sorensen, D. M. Kim, J. J. Nielsen, and P. Popovski, "Analysis of Latency and MAC-Layer Performance for Class A LoRaWAN," IEEE Wirel. Commun. Lett., vol. 6, no. 5, pp. 566-569, 2017.

[8] J. M. Marais, A. M. Abu-Mahfouz, and G. P. Hancke, "A Survey on the Viability of Confirmed Traffic in a LoRaWAN," IEEE Access, vol. 8, pp. 9296-9311, 2020, doi: 10.1109/ACCESS.2020.2964909. 
[9] M. He, S. Cheng, T. Ma, and S. Lv, “A Bit Error Rate Optimization Method for WSN Node Energy Consumption," 2020, pp. 84-95.

[10] B. Su, Z. Qin, and Q. Ni, "Energy Efficient Uplink Transmissions in LoRa Networks," IEEE Trans. Commun., vol. 68, no. 8, pp. 4960-4972, 2020.

[11] B. Paul, "A Novel Energy-Efficient Routing Scheme for LoRa Networks," IEEE Sens. J., vol. 20, no. 15, pp. 8858-8866, 2020.

[12] M. Nurgaliyev, A. Saymbetov, Y. Yashchyshyn, N. Kuttybay, and D. Tukymbekov, "Prediction of energy consumption for LoRa based wireless sensors network," Wirel. Networks, vol. 26, 2020, doi: 10.1007/s11276-02002276-5.

[13] Y. Song, J. Lin, M. Tang, and S. Dong, "An Internet of Energy Things Based on Wireless LPWAN," Engineering, vol. 3, no. 4, pp. 460-466, 2017, doi: https://doi.org/10.1016/J.ENG.2017.04.011.

[14] C. Tunc and N. Akar, "Markov fluid queue model of an energy harvesting IoT device with adaptive sensing," Perform. Eval., vol. 111, pp. 1-16, 2017, doi: https://doi.org/10.1016/j.peva.2017.03.004.

[15] Semtech Corporation, "LoRa Modulation Basics," 2015. http://www.semtech.com/images/datasheet/an1200.2 2.pdf,. (Access Date: 25 August, 2020).

[16] T. Bouguera, J. F. Diouris, J. J. Chaillout, R. Jaouadi, and G. Andrieux, "Energy Consumption Model for Sensor Nodes Based on LoRa and LoRaWAN," Sensors (Basel), vol. 18, no. 7, p. 2104, 2018, doi: doi:10.3390/s18072104.

[17] L. Casals, B. Mir, R. Vidal, and C. Gomez, "Modeling the Energy Performance of LoRaWAN," Sensors (Basel), vol. 17, no. 10, p. 2364, 2017, doi:10.3390/s17102364.

[18] T. Deng, J. Zhu, and Z. Nie, "An improved LoRaWAN protocol based on adaptive duty cycle," in 2017 IEEE 3rd Information Technology and Mechatronics Engineering Conference (ITOEC), 2017, pp. 1122-1125.

[19] A. Lavric, A. I. Petrariu, and V. Popa, "Long Range SigFox Communication Protocol Scalability Analysis Under Large-Scale, High-Density Conditions," IEEE Access, vol. 7, pp. 35816-35825, 2019.

[20] Y. Jaradat, M. Masoud, I. Jannoud, and D. Zaidan, "The Impact of Nodes Distribution on Energy Consumption in WSN," in 2019 IEEE Jordan International Joint Conference on Electrical Engineering and Information Technology (JEEIT), 2019, pp. 590-595.

[21] LoRa Alliance ${ }^{\circledR}$, "LoRa Alliance - LoRaWAN," 2020. https://lora-alliance.org/ (Access Date: 30 August, 2020).

[22] T. Elshabrawy and J. Robert, "The Impact of ISM Interference on LoRa BER Performance," in 2018 IEEE Global Conference on Internet of Things (GCIoT), 2018, pp. 1-5.
[23] R. Sanchez-Iborra and M.-D. Cano, "State of the art in LP-WAN solutions for industrial IoT services," Sensors, vol. 16, p. 708, 2016, doi: 10.3390/s16050708.

[24] D. M. Mount, “CMSC 754 - Computational Geometry. Lecture Notes., 2002. http://www.cs.umd.edu/ $\sim$ mount/754/Lects/754lects.pdf. (Access Date: 8 August, 2020).

[25] B. A. da Silva and R. M. de Moraes, "Consumo de Energia em Função da Taxa de Transmissão e do Tamanho do Pacote em Redes Ad Hoc Aloha de um Salto," in Anais do XVII Workshop em Desempenho de Sistemas Computacionaise de Comunicação, 2018, doi:10.5753 /wperformance.2018.3321. 